Referencia para citar este artículo: Hernández, J.; Cardona-Arango, D., \& Segura-Cardona, A. M. (2018). Construcción y análisis de un índice de vulnerabilidad social en la población joven. Revista Latinoamericana de Ciencias Sociales, Niñez y Juventud, 16(1), 403-412. doi:10.11600/1692715x.16125

\title{
Construcción y análisis de un índice de vulnerabilidad social en la población joven*
}

\author{
JONATHAN HERNÁNDEZ ${ }^{* *}$ \\ Profesor Institución Universitaria de Envigado, Colombia. \\ DORIS CARDONA-ARANGO ${ }^{* * *}$ \\ Investigador Universidad CES, Colombia. \\ ÁNGELA M. SEGURA-CARDona**** \\ Investigador Universidad CES, Colombia.
}

\section{Artículo recibido en enero 31 de 2017; artículo aceptado en mayo 2 de 2017 (Eds.)}

- Resumen (analítico): En esta investigación nos trazamos como objetivo explorar la vulnerabilidad social en la población entre los 15 y 24 años de edad, en Medellín; para ello diseñamos un índice usando la técnica de análisis factorial con variables contenidas en la Encuesta de Calidad de Vida de la ciudad, en el año 2014. Encontramos que la población joven con alta vulnerabilidad, en su mayoría es población masculina, con educación básica, conformada por mestizos, indígenas o raizales que viven en estrato socioeconómico 2 (bajo). El estudio cuenta con limitaciones para inferir los resultados a toda la población joven de la ciudad de Medellín; sin embargo, aporta información útil para identificar las características de los individuos jóvenes altamente vulnerables.

Palabras clave: joven, grupo vulnerable, calidad de vida, acceso a la educación, análisis factorial (Tesauro de Ciencias Sociales de la Unesco).

Palabra clave autores: construcción de índice.

\section{Construction and analysis of a social vulnerability index in young population}

- Abstract (analytical): the research aimed to explore the social vulnerability in the population between 15 and 24 years old; an index was designed using a factorial analysis technique with variables included in the Quality of Life Survey of the Municipality of Medellin, in 2014. It was identified that young people with high vulnerability were a primarily male population with a basic education level, identified as mestizos, indigenous or from afro-descendent and raizal communities and living at a socioeconomic level 2 (low). The study has limitations in inferring the results to cover all young people in the city of Medellin; however, it does provide useful information to identify the characteristics of highly vulnerable young people.

\footnotetext{
* Este artículo de investigación científica y tecnológica presenta resultados de la investigación denominada, "Vulnerabilidad social en la población joven. Medellín 2014", presentada por el autor principal para optar el título de Magíster en Epidemiología en la Universidad CES. Realizada entre Agosto de 2015 y Mayo de 2016. Acta139, Proy. 019. Área del Conocimiento: Ciencias Sociales. Sub-área: Demografía.

** Psicólogo. Magíster en Epidemiología. Profesor Facultad de Psicología Institución Universitaria de Envigado. Correo electrónico: nathancalle@yahoo.es

*** Doctora en Demografía. Grupo de Investigación Epidemiología y Bioestadística, Universidad CES. Orcid: 0000-0003-4338-588X. Índice H5: 15. Correo electrónico: dcardona@ces.edu.co

**** Doctora en Epidemiología. Grupo de Investigación Epidemiología y Bioestadística, Universidad CES. Orcid: 0000-0002-0010-1413. Índice H5: 11. Correo electrónico: asegura@ces.edu.co
} 
Key words: young, vulnerable group, quality of life, access to education, factorial analysis (Social Science Unesco Thesaurus).

Authors key word: construction of index.

\section{Construção e análise do índice de vulnerabilidade social na população jovem}

- Resumo (analítico): o objetivo da pesquisa foi explorar a vulnerabilidade social na população numa faixa etária de15 a 24 anos, para isso foi desenhado um índice utilizando a técnica de análises fatorial com variáveis contidas no questionário de qualidade de vida da cidade de Medellín no ano 2014. Encontrou-se que a população jovem com alta vulnerabilidade foi principalmente a população masculina, com ensino fundamental, identificados como mestiços, indígenas ou nativos que viviam em nível socioeconómico 2 (baixo). O estudo tem as limitações para deduzir os resultados para toda a população jovem da cidade de Medellín, mesmo assim, fornece informação útil para identificar as características dos jovens com alta vulnerabilidade.

Palavras chave: jovem, grupo vulnerável, qualidade de vida, acesso à educação, análises fatorial (Thesaurus de Ciências Sociais da Unesco).

Palavras-chave do autor: construção de índice.

\section{-1. Introducción. -2. Materiales y métodos. -3. Resultados. -4. Discusión. -5. Conclusión. -Lista de referencias.}

\section{Introducción}

La vulnerabilidad social es un concepto multidimensional que surge en las ciencias sociales y humanas como una propuesta para superar el enfoque tradicional de pobreza centrado en la insuficiencia de ingresos, siendo un enfoque limitado para comprender las condiciones de vida de las poblaciones más desamparadas (Castro \& Cano, 2013). González (2009) expone que la vulnerabilidad social es el conjunto de limitaciones o desventajas que las personas encuentran para acceder y usar los activos que se distribuyen en las sociedades. Kaztman y Filgueira, desde su enfoque "Activos, vulnerabilidad y estructura de oportunidades" (Aveo), clasifican los activos en capital físico, humano, financiero o productivo, y social; según los autores, los individuos tienen tres fuentes principales de capitales: el Estado, el mercado y la comunidad (Kaztman, 1999).

El capital físico está constituido por recursos tangibles que a nivel del hogar incluyen las condiciones de la vivienda y los bienes durables de consumo (Kaztman, 1999). Este capital constituye una de las dimensiones fundamentales del modelo Aveo, puesto que un individuo inmerso en un hábitat $\mathrm{y}$ en una vivienda precaria percibe como limitadas sus posibilidades de desarrollo y sano crecimiento. Para operacionalizar esta dimensión de la vulnerabilidad, comúnmente se utilizan variables indicativas referidas al tipo de vivienda, propiedad de la misma, hacinamiento, saneamiento, infraestructura y equipamiento (Golovanevsky, 2007).

En el capital humano se analizan aspectos como la salud y la educación, puesto que representan condiciones esenciales para las personas; por ejemplo, la desigualdad en el acceso a los servicios de salud en países pobres se asocia a problemas poblacionales como la mortalidad materna e infantil atribuible a causas evitables. De otra parte, la relevancia de la educación radica en que se vincula con la formación de activos, al posibilitar una inserción laboral más sólida y un flujo de ingresos más confiable (Golovanevsky, 2007).

Para el análisis de la situación de salud se consideran aspectos como la natalidad, la salud sexual y reproductiva, y los recursos disponibles para la atención en salud, en tanto en lo educativo se toman como variables aquellas vinculadas a la asistencia escolar, a la deserción y a los niveles de instrucción alcanzados (Golovanevsky, 2007).

El capital financiero o productivo constituye el concepto más antiguo de capital, 
al estar constituido por los recursos monetarios disponibles en cada hogar para acceder a los bienes de consumo (Kaztman, 1999). En contraste, el capital social es el concepto más novedoso de capital y, por lo tanto, no existe aún consenso acerca de su definición; según Kaztman (1999), el capital social consiste en un conjunto de reglas, normas y obligaciones, dentro de las relaciones, estructuras y arreglos institucionales sociales que promueven la reciprocidad y la confianza. Ahora bien, con el fin de abordar su medición se incluyen en el capital social variables relacionadas con el ámbito comunitario, como la asistencia a grupos religiosos, la participación en actividades comunales, la inscripción en clubes deportivos, las condiciones de seguridad, etc. (Arora, Dheeraj, \& Piyush, 2015).

La población joven es aquella que se ubica generalmente entre los 15 y los 24 años de edad, y cuenta con capitales potenciales que favorecen el desarrollo de los países (Unicef, 2011); sin embargo, su realidad está marcada por la precariedad y la exclusión, especialmente la de quienes viven en estratos socioeconómicos bajos. La incapacidad de las familias para invertir en la educación de los hijos e hijas, las exigencias del mercado laboral que excluye a los individuos jóvenes de las posiciones laborales mejor remuneradas por el nivel formativo, las altas tasas de fecundidad que contribuyen al ciclo de pobreza, y la exposición a situaciones de violencia, generan en este grupo poblacional condiciones de alta vulnerabilidad social (Arora et al., 2015).

La Organización Internacional del Trabajo (OIT) reporta que en América Latina cerca de 2,8 millones de jóvenes no estudian ni trabajan, y que la proporción de jóvenes en desempleo es el triple en comparación con la población adulta, hallándose de cada 10 sujetos jóvenes ocupados seis en condiciones de informalidad, con bajos ingresos, inestabilidad laboral y carencia de protección y derechos (OIT, 2013).

La población joven colombiana de los sectores más pobres no es ajena a las problemáticas enunciadas de falta de oportunidades educativas y laborales; adicionalmente experimenta marginación a nivel del capital social para acceder a la participación política, a la recreación y a las posibilidades de expresión (Muñoz, 2003).

En el contexto de Medellín, en el año 2011 se construyó un índice de desarrollo juvenil (IDJM), mediante el cual se evidenció cómo el desempleo, la informalidad y la inestabilidad laboral afectaba el desarrollo social de la población joven en la ciudad, mientras que condiciones como el acceso a los servicios de salud y a la tecnología favorecían su desarrollo. A partir de este índice se recomendó revisar continuamente la situación de la población joven en la ciudad con el fin de proponer acciones para mejorar sus condiciones de vida (Grisales, Márquez, \& Rojas, 2014).

Partiendo de lo anterior, en el presente estudio construimos un índice para explorar la vulnerabilidad social en la población joven de la ciudad de Medellín, según las características individuales y las condiciones socioeconómicas de los sujetos jóvenes.

\section{Materiales y métodos}

Este trabajo es un estudio transversal exploratorio de fuente de información secundaria. Utilizamos 8406 registros correspondientes a la población de edades entre 15 y 24, que respondieron la Encuesta de Calidad de Vida (ECV) del municipio de Medellín en el año 2014; la base de datos fue otorgada por el Departamento Administrativo de Planeación Municipal a la Universidad CES. Excluimos a los jóvenes con información incompleta en la base de datos, en las variables de interés para la investigación.

Siguiendo el modelo teórico propuesto por Kaztman y Filgueira para la construcción del índice de vulnerabilidad social, seleccionamos inicialmente ciento cuatro variables de la ECV del año 2014, 49 de las cuales respondían al capital físico, 17 al capital humano, 16 al capital financiero o productivo y 22 al capital social.

Aplicamos cuantificación óptima a las variables cualitativas y utilizamos un análisis factorial seleccionando como método de extracción el análisis de componentes principales (ACP) con rotación varimax, técnica que se ha reportado en la construcción de índices sociales (Cardona-Arango, Agudelo, 
Restrepo, \& Segura-Cardona, 2014; Grisales et al., 2014). Además, para evaluar la pertinencia del análisis factorial tuvimos en cuenta un valor de $\mathrm{p}<0,05$ en la prueba de esfericidad de Bartlett, y una adecuación muestral de KaiserMeyer-Olkin $(\mathrm{KMO}) \geq 0,5$.

Mediante la técnica de análisis factorial redujimos a un número de ocho variables, con las cuales calculamos cuatro componentes principales de la vulnerabilidad social: satisfacción de las necesidades de subsistencia (capital humano y capital financiero $\mathrm{o}$ productivo), inversión en educación y esparcimiento (capital financiero o productivo y capital físico), protección social (capital humano y capital social), y ocio y tiempo libre (capital físico y capital social).

Figura 1. Componentes principales de la vulnerabilidad social en población joven.

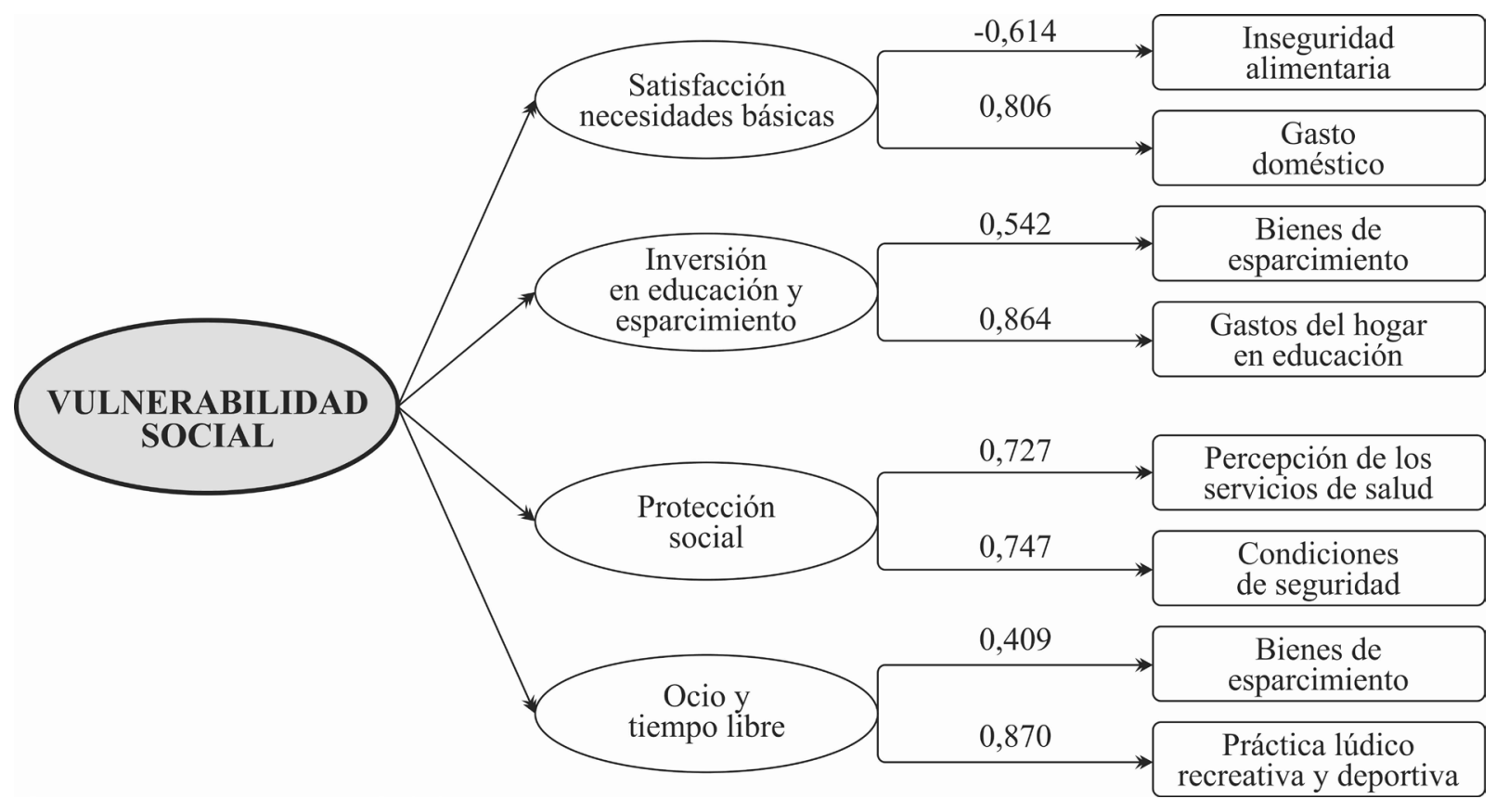

El modelo de vulnerabilidad social obtuvo un valor de $p=0,000$ en la prueba de esfericidad de Bartlett, y una puntuación en el índice de adecuación muestral de Kaiser-Meyer-Olkin $(\mathrm{KMO})=0,515$; lo que indica la pertinencia del análisis factorial realizado; además, el modelo cumplió con el principio de parsimonia, presentando la mayor variabilidad explicada con el menor número de variables.

Con las puntuaciones factoriales que obtuvieron los individuos en cada uno de los cuatro componentes calculados, es decir, tomando los valores de los factores en cada observación, construimos la escala de vulnerabilidad social en tres grados:

- Alta vulnerabilidad: personas que registraron tres o cuatro puntuaciones con signo negativo.
- Mediana vulnerabilidad: personas que registraron una o dos puntuaciones con signo negativo.

- Baja vulnerabilidad: personas que registraron en los cuatro componentes puntuaciones con signo positivo.

Con la escala construida buscamos relaciones no causales entre las características individuales y socioeconómicas y los tres grados de vulnerabilidad social, usando la prueba Chi-cuadrado. Los datos fueron procesados utilizando el paquete estadístico SPSS ${ }^{\mathrm{TM}}$ versión 21, licenciado por la Universidad CES.

\section{Resultados}

Según el índice de vulnerabilidad social, el 9,5\% (798) de la población entre 15 y 24 
años de edad que respondió la ECV, presentó en el año 2014 baja vulnerabilidad, el 65,8\% (5528) mediana vulnerabilidad, y el 19,7\% (1660) alta vulnerabilidad. Al analizar el grado de vulnerabilidad según características individuales, el 53,2\% de la población joven con alta vulnerabilidad tenía entre 15 y 19 años de edad, mientras que en los grados de baja y mediana vulnerabilidad predominaba la población entre 20 y 24 años. Por sexo, identificamos que el $53 \%$ de los jóvenes que registraron alta vulnerabilidad eran hombres, con un mayor número de población femenina en los otros dos grados de vulnerabilidad social.
En el grado de alta vulnerabilidad apreciamos un alto porcentaje de jóvenes con educación básica, mientras que en el grado de baja y mediana vulnerabilidad encontramos una proporción mayor de población con educación media. En cuanto a la etnia, hallamos que los individuos mestizos, indígenas o raizales representaban más de la mitad de la población en los tres grados de vulnerabilidad. Conforme a lo anterior observamos una asociación estadísticamente significativa entre el rango de edad, el sexo, el nivel de escolaridad y la etnia, con el grado de vulnerabilidad social en la población joven $(\mathrm{p}<0,05)$.

Tabla 1. Características individuales de los individuos jóvenes entre 15 y 24 años de edad, por grado de vulnerabilidad. Medellín, 2014.

\begin{tabular}{|c|c|c|c|c|c|c|}
\hline & & o de vulneral & dad & & & \\
\hline Variable & $\begin{array}{l}\text { Baja } \\
\text { n (\%) }\end{array}$ & $\begin{array}{c}\text { Mediana } \\
\text { n (\%) }\end{array}$ & $\begin{array}{c}\text { Alta } \\
\text { n (\%) }\end{array}$ & $\mathbf{n}$ & $\mathbf{X}^{2}$ & Valor $\mathbf{p}$ \\
\hline Edad & & & & & $33,607 *$ & 0,000 \\
\hline Entre 15 y 19 años & $377(47,2)$ & $2493(45,1)$ & $883(53,2)$ & 3753 & & \\
\hline Entre 20-24 años & $421(52,8)$ & $3035(54,9)$ & $777(46,8)$ & 4233 & & \\
\hline Sexo & & & & & 21,800 & 0,000 \\
\hline Hombres & $351(44,0)$ & $2631(47,6)$ & $880(53,0)$ & 3862 & & \\
\hline Mujeres & $447(56,0)$ & $2897(52,4)$ & $780(47,0)$ & 4124 & & \\
\hline Nivel de escolaridad & & & & & 19,445 & 0,003 \\
\hline Ninguno & $12(1,6)$ & $73(1,4)$ & $24(1,5)$ & 109 & & \\
\hline Básica & $355(46,5)$ & $2289(44,3)$ & $762(48,8)$ & 3406 & & \\
\hline Media & $366(47,9)$ & $2503(48,4)$ & $716(45,9)$ & 3585 & & \\
\hline Superior & $31(4,1)$ & $302(5,8)$ & $59(3,8)$ & 392 & & \\
\hline Etnia & & & & & 9,740 & 0,045 \\
\hline Sujetos Blancos & $187(23,9)$ & $1104(20,2)$ & $320(19,6)$ & 1611 & & \\
\hline Sujetos Afrodescendientes & $33(4,2)$ & $183(3,4)$ & $66(4,0)$ & 282 & & \\
\hline Otro (sujetos mestizos) & $563(71,9)$ & $4166(76,4)$ & $1252(76,4)$ & 5981 & & \\
\hline
\end{tabular}

Nota. $*$ Sig. Estadística valor $\mathrm{p}<0,05$

En los tres grados de vulnerabilidad predominaron los individuos jóvenes que habitaban en el estrato socioeconómico 2, quienes representaron el 38,4\%, el 39,4\%, y el $45 \%$ del total de la población con alta, mediana y baja vulnerabilidad social, respectivamente. 
Según las zonas de residencia en la ciudad de Medellín, los jóvenes y las jóvenes con alta vulnerabilidad se encontraron especialmente en las zonas Nororiental, Noroccidental y Centroccidental. De otro lado, la zona Centroriental tuvo una mayor proporción de jóvenes con baja y mediana vulnerabilidad, en comparación con la zona Centroccidental. Por otra parte, observamos que la mayoría de los sujetos jóvenes con alta vulnerabilidad reportaron que en sus viviendas habitaban entre cuatro y nueve personas. Como resultado de lo descrito encontramos una asociación estadísticamente significativa entre el estrato socioeconómico, la zona de residencia, y el número de personas en el hogar con el grado de vulnerabilidad social en la población joven $(\mathrm{p}<0,05)$.

Tabla 2. Condiciones socioeconómicas de los jóvenes entre 15 y 24 años de edad, por grado de vulnerabilidad. Medellín, 2014.

\begin{tabular}{|c|c|c|c|c|c|c|}
\hline \multirow[b]{2}{*}{ Variable } & \multicolumn{3}{|c|}{ Grado de vulnerabilidad } & \multirow[b]{2}{*}{$\mathbf{n}$} & \multirow[b]{2}{*}{$\mathbf{X}^{2}$} & \multirow[b]{2}{*}{ Valor $\mathbf{p}$} \\
\hline & $\begin{array}{l}\text { Baja } \\
\text { n (\%) }\end{array}$ & $\begin{array}{c}\text { Mediana } \\
\text { n (\%) }\end{array}$ & $\begin{array}{c}\text { Alta } \\
\text { n (\%) }\end{array}$ & & & \\
\hline Estrato socioeconómico** & & & & & 31,494 & $0,000 *$ \\
\hline Estrato 1 (bajo) & $124(15,5)$ & $909(16,4)$ & $324(19,5)$ & 1357 & & \\
\hline Estrato 2 (bajo-bajo) & $359(45,0)$ & $2177(39,4)$ & $637(38,4)$ & 3173 & & \\
\hline Estrato 3 (medio bajo) & $212(26,6)$ & $1560(28,2)$ & $437(26,3)$ & 2209 & & \\
\hline Estrato 4 (medio) & $52(6,5)$ & $432(7,8)$ & $158(9,5)$ & 642 & & \\
\hline Estrato 5 (medio alto) & $33(4,1)$ & $287(5,2)$ & $67(4,0)$ & 387 & & \\
\hline Estrato 6 (alto) & $18(2,3)$ & $163(2,9)$ & $37(2,2)$ & 218 & & \\
\hline Zona de residencia & & & & & 52,496 & $0,000 *$ \\
\hline 1 Nororiental & $204(25,6)$ & $1435(26,0)$ & $479(28,9)$ & 2118 & & \\
\hline 2 Noroccidental & $171(21,4)$ & $1144(20,7)$ & $362(21,8)$ & 1677 & & \\
\hline 3 Centroriental & $129(16,2)$ & $878(15,9)$ & $255(15,4)$ & 1262 & & \\
\hline 4 Centroccidental & $92(11,5)$ & $795(14,4)$ & $257(15,5)$ & 1144 & & \\
\hline 5 Suroriental & $22(2,8)$ & $207(3,7)$ & $60(3,6)$ & 289 & & \\
\hline 6 Suroccidental & $78(9,8)$ & $538(9,7)$ & $158(9,5)$ & 774 & & \\
\hline Zona rural & $102(12,8)$ & $531(9,6)$ & $89(5,4)$ & 722 & & \\
\hline Número de personas en el hogar & & & & & 17,968 & $0,001 *$ \\
\hline Entre 1 y 3 & $229(28,7)$ & $1505(27,2)$ & $373(22,5)$ & 2107 & & \\
\hline Entre 4 y 9 & $553(69,3)$ & $3905(70,6)$ & $1244(74,9)$ & 5702 & & \\
\hline Mayor o igual a 10 & $16(2,0)$ & $118(2,1)$ & $43(2,6)$ & 177 & & \\
\hline
\end{tabular}

Nota. *Sig. Estadística valor $\mathrm{p}<0,05$

**Clasificación de la vivienda según el Departamento Nacional de Estadística (Dane).

Al discriminar la población joven con alta vulnerabilidad por comunas de residencia, registramos en las comunas 7 (Robledo) y 13 (San Javier) la mayor prevalencia, seguidas de las comunas 1 (Popular), 2 (Santa Cruz),
3 (Manrique), 4 (Aranjuez), 5 (Castilla) y 6 (Doce de Octubre), y las de menor prevalencia fueron los corregimientos y las comunas $10(\mathrm{La}$ Candelaria) y 15 (Guayabal) (ver figura 2). 
Figura 2. Distribución espacial de la población entre 15 y 24 años de edad con alta vulnerabilidad, según comuna de residencia. Medellín, 2014.

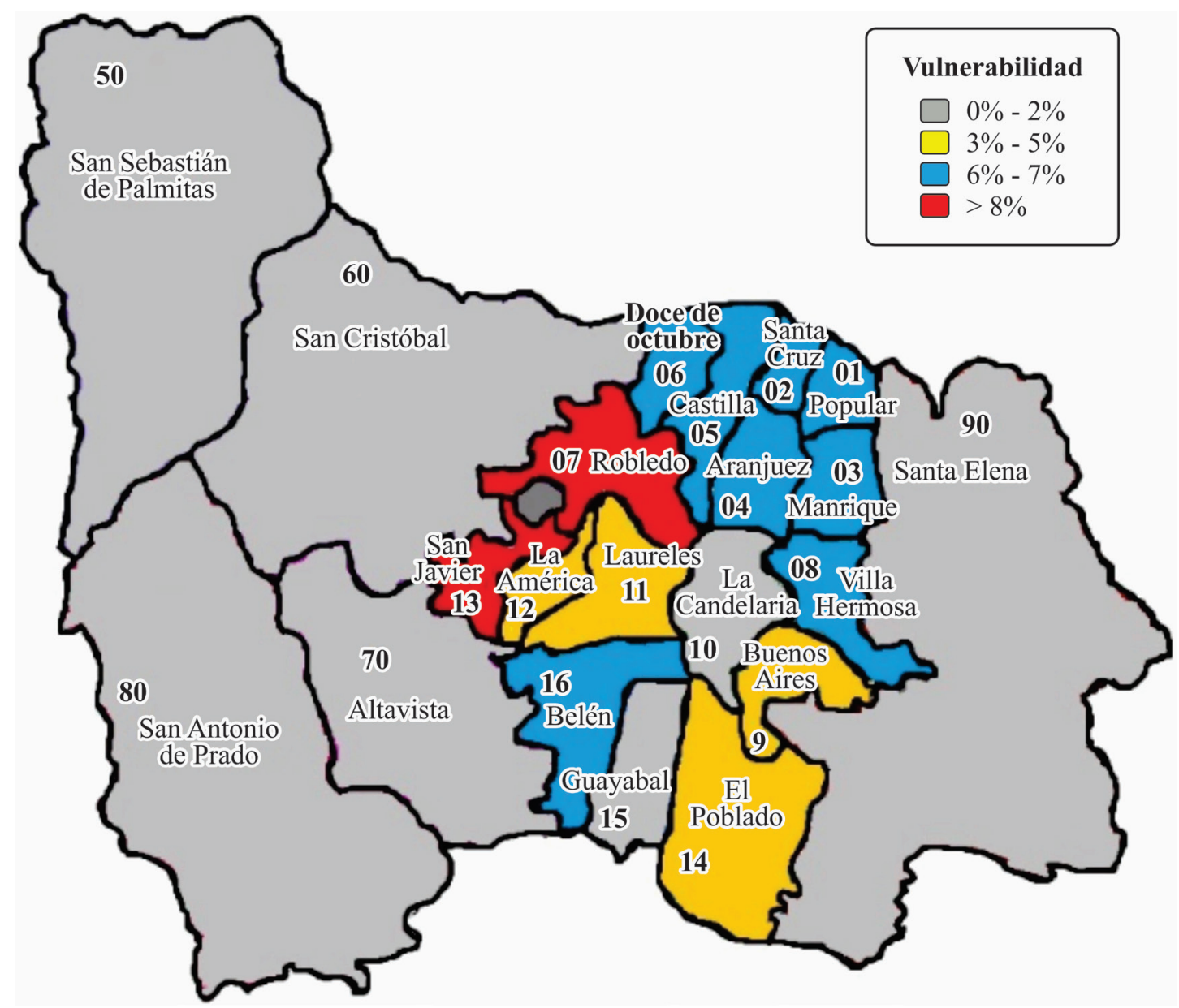

\section{Discusión}

Desde comienzos del siglo XX las personas jóvenes se han considerado como promesa de transformación social, económica, política y cultural de las sociedades, radicando aquí la importancia de su abordaje desde diferentes disciplinas académicas y desde el sector político (Brito, 1998). No obstante, las sociedades en vía de desarrollo como Colombia, no siempre brindan las oportunidades para que la población joven pueda acceder a bienes y servicios que les posibiliten mejorar su calidad de vida, lo que pone a esta población en una situación de alta vulnerabilidad social (Rodríguez, 2001).

Partiendo de la teoría propuesta por Kaztman y Filgueira, denominada "Activos, vulnerabilidad y estructura de oportunidades
(Aveo)" (Kaztman, 1999), construimos un índice de vulnerabilidad social en la población joven de la ciudad de Medellín (Colombia), cuyos componentes principales fueron: satisfacción de las necesidades de subsistencia, inversión en educación y esparcimiento, protección social, y ocio y tiempo libre. Estos componentes están en consonancia con los hallazgos de las Naciones Unidas en 2009 en los países de Argentina, Brasil, Uruguay y Paraguay, donde encontramos que los jóvenes y las jóvenes presentaban vulnerabilidad social a partir de un déficit en las dimensiones de salud y saneamiento, acceso a la educación, ingresos, condiciones de vivienda, y protección social.

Al observar el grado de vulnerabilidad social por rango de edad encontramos que los individuos jóvenes de edades entre 15 y 19 
años, representaron la mayoría de la población con alta vulnerabilidad. A propósito, Osorio y Hernández (2011) hallaron en Colombia que la población de ese rango de edad está expuesta a condiciones que afectan su desarrollo social, tal como la deserción escolar; y la OIT (2013), ha revelado que en Latinoamérica los sujetos jóvenes de esas edades son más proclives a trabajos informales bajo condiciones desfavorables.

En el estudio, los hombres registraron la mayor proporción en el grado de alta vulnerabilidad social, mientras las mujeres predominaron en los grados de baja y mediana vulnerabilidad. Estos resultados pueden ser explicados, dado que en Colombia se ha observado que los hombres son quienes menos invierten en educación; según el Departamento Administrativo Nacional de Estadística (Dane) (2005), la proporción de mujeres con educación media es del $4,8 \%$ frente a un $4,3 \%$ en los hombres, en tanto el porcentaje de mujeres profesionales es del 7,0\%, mientras que en los hombres corresponde al $6,7 \%$. Además, los hombres son quienes menos buscan protección social, ya que su asistencia a los servicios de salud es más baja en comparación con el sexo opuesto (Pinilla, Forero, \& Valdivieso, 2009).

En cuanto a lo educativo, en los jóvenes y las jóvenes con alta vulnerabilidad predominó la educación básica; según Eckert (2006), en las sociedades actuales del conocimiento los jóvenes sin educación suficiente tienen pocas oportunidades para acceder al mercado laboral, situación que los pone en una condición de vulnerabilidad social al afectarse la satisfacción de sus necesidades básicas.

Otra característica individual en la población joven, que asociamos con la vulnerabilidad social, fue la etnia, lo que está en conformidad con los estudios de la Cepal, en los cuales se ha reportado en América Latina y el Caribe racismo y discriminación frente a grupos étnicos como los afrodescendientes e indígenas, lo que ha traído pobreza, exclusión y desigualdad, haciendo vulnerables estos grupos en los país en desarrollo (Bello \& Rangel, 2002).

Analizando las condiciones socioeconómicas de la gente joven en los estratos 1 (bajo-bajo), 2 (bajo) y 3 (medio bajo), la proporción de alta vulnerabilidad fue mayor que en los estratos altos, lo que es explicable dado que los individuos jóvenes de estratos socioeconómicos bajos presentan mayores barreras para acceder a bienes y servicios. En un estudio previo, encontramos que los sujetos jóvenes con escasos recursos económicos en la ciudad de Medellín presentan un déficit en los componentes de inversión en educación y esparcimiento, así como en ocio y tiempo libre, ya que el acceso a la tecnología en esta población es inferior en comparación con los del estrato socioeconómico alto, y muchos no acceden a eventos culturales principalmente porque su capacidad económica se los impide (Grisales et al., 2014).

Los jóvenes y las jóvenes con alta vulnerabilidad social se encontraron principalmente en las zonas 1 Nororiental, 2 Noroccidental y 4 Centroccidental de la ciudad de Medellín; en otros estudios se ha dado a conocer que en estas zonas se presentan problemáticas como el consumo de sustancias psicoactivas (Torres, 2012) y el accionar de grupos al margen de la ley (Vargas \& García, 2008), que afectan el componente de protección social en las personas jóvenes por las condiciones de seguridad en el contexto que les rodea.

Además del estrato socioeconómico y de la zona de residencia, el número de personas en el hogar también influye sobre el grado de vulnerabilidad social, lo que podría sugerir que la satisfacción de las necesidades básicas, al igual que otros componentes, pueden verse afectados por el número de personas en la vivienda; por ejemplo, en la literatura se ha reportado que las condiciones de hacinamiento traen consecuencias sobre la salud y el comportamiento de las personas integrantes del hogar (Rojas, 2004).

Los anteriores hallazgos confirman que, para analizar la vulnerabilidad social en los individuos jóvenes, es necesario centrarse en los capitales que posee esta población para hacer frente a las demandas cotidianas, los cuales son generados por una estructura de oportunidades desde el Estado, el mercado y la sociedad. Una de las consecuencias de los sistemas capitalistas 
actuales es precisamente el incremento de la vulnerabilidad social -especialmente en países en desarrollo-, lo que se ha convertido en un reto para las políticas sociales tendientes a mejorar la calidad de vida de las poblaciones más vulnerables (Rodríguez, 2001).

Aunque el presente estudio presentó como limitación que la Encuesta de Calidad de Vida del año 2014 no fue aplicada a una muestra representativa de la población joven, puesto que el muestreo se realizó por estrato socioeconómico, comuna y corregimiento, dado el tamaño de la muestra de 8406 jóvenes, es factible tomar decisiones con los resultados presentados enfocando las intervenciones psicosociales en los componentes que aportan a la vulnerabilidad social de los sujetos jóvenes, teniendo en cuenta las zonas de la ciudad de Medellín donde encontramos la población con mayor vulnerabilidad.

Otra limitación del estudio fue el insuficiente desarrollo de investigaciones en el contexto, en las que se haya analizado la vulnerabilidad social de la población joven desde un enfoque cuantitativo con análisis multivariado, tomando como modelo teórico el propuesto por Kaztman y Filgueira. A propósito de lo anterior, Grisales et al. (2014) también habían hecho mención a la falta de estudios encaminados a calcular el desarrollo juvenil cuando iniciaron su proyecto de investigación.

Dada esta limitación, incluimos en la discusión investigaciones que no fueron de reciente publicación, pero que abordan la población joven en uno o varios de los componentes de la vulnerabilidad social identificados en el estudio.

\section{Conclusión}

La vulnerabilidad social de la población joven es un fenómeno multidimensional; en esa línea, encontramos que en las personas jóvenes de la ciudad de Medellín que respondieron la ECV, la vulnerabilidad social se presenta según la satisfacción de las necesidades de subsistencia, la inversión en educación y esparcimiento, la protección social y el ocio y el tiempo libre. Con estos componentes construimos un índice de vulnerabilidad social y encontramos relaciones no causales con la edad, el sexo, el nivel educativo, la etnia, el estrato socioeconómico, la zona de residencia y el número de personas en el hogar.

\section{Lista de referencias}

Arora, S., Dheeraj, S., \& Piyush, G. (2015). Defining and measuring vulnerability in young people. Indian Journal of Community Medicine: Official publication of Indian Association of Preventive \& Social Medicine, 40(3), 193-197. Recuperado de: http://www.ncbi.nlm.nih.gov/pmc/ articles/PMC4478662/. doi:10.4103/09700218.158868

Bello, A., \& Rangel, M. (2002). La equidad y la exclusión de los pueblos indígenas y afrodescendientes en América Latina y el Caribe. Revista Cepal, (76), 39-54. Recuperado de: http://repositorio.cepal.org/bitstream/ handle/11362/10800/1/076039054_es.pdf.

Brito, R. (1998). Hacia una sociología de la juventud. Algunos elementos para la deconstrucción de un nuevo paradigma. Última Década, (9), 1-7. Recuperado de: $\quad$ http://www.redalyc.org/articulo. oa? id=19500909.

Cardona-Arango, D., Agudelo-Martínez, A., Restrepo-Molina, L., \& Segura-Cardona, A. M. (2014). Índice de vulnerabilidad de la población en situación de discapacidad en Medellín. Revista Salud Pública, 16(1), 1-12. doi: https://doi.org/10.15446/rsap. v16n1.23110

Castro, L. I., \& Cano, R. (2013). Pobreza y vulnerabilidad: factores de riesgo en el proceso educativo. Revista Contextos Educativos, (16), 55-62. Recuperado de: https://publicaciones.unirioja.es/ojs/index. $\mathrm{php} /$ contextos/article/view/1290.

Departamento Administrativo Nacional de Estadística (Dane). (2005). Censo General 2005. Datos desagregados por sexo. Recuperado de: http://www.dane.gov.co/ files/censo2005/gene 1503 07.pdf

Eckert, H. (2006). Entre el fracaso escolar y las dificultades de inserción profesional: la vulnerabilidad de los jóvenes sin 
formación en el inicio de la sociedad del conocimiento. Revista de Educación (341), 35-56. Recuperado de: http://www. revistaeducacion.mec.es/re341/re341_02.p Golovanevsky, L. (2007). Vulnerabilidad social: una propuesta para su medición en Argentina. Revista de Economía y Estadística, 45(2), 53-94. Recuperado de: https://revistas.unc.edu.ar/index.php/ REyE/article/viewFile/3840/6780

González, L. (2009). Lecturas sobre vulnerabilidad y desigualdad social. Córdoba:Universidad Nacional deCórdoba. Recuperado de: http://209.177.156.169/ libreria_cm/archivos/pdf_414.pdf

Grisales, H., Márquez, F., \& Rojas, J. (2014). Construcción de un índice de desarrollo para jóvenes de 14 a 26 años, Medellín, Colombia. Revista Panamericana de Salud Pública, 35(4), 242-247. Recuperado de: http://www.scielosp.org/pdf/rpsp/ v35n4/02.pdf

Kaztman, R. (1999). Activos y estructuras de oportunidades: estudio sobre las raices de la vulnerabilidad social en Uruguay. Santiago de Chile: Comisión Económica para América Latina y el Caribe. Recuperado de: http://repositorio. cepal.org/bitstream/handle/11362/28651/ LCmvdR180 es.pdf? sequence $=1$

Muñoz, G. (2003). Temas y problemas de los jóvenes colombianos al comenzar el siglo XXI. Revista Latinoamericana de Ciencias Sociales, Niñez y Juventud, 1(1), 145-180. Recuperado de: http:// revistaumanizales.cinde.org.co/index.php/ RevistaLatinoamericana/article/view/337

Organización Internacional del Trabajo (OIT). (2013). Trabajo decente y juventud en América Latina: políticas para la acción. Recuperado de: http://www.ilo.org/ americas/publicaciones/WCMS_235577/ lang--es/index.htm.

Osorio, P., \& Hernández, M. (2011). Prevalencia de deserción escolar en embarazadas adolescentes de instituciones educativas oficiales del Valle del Cauca, Colombia 2006. Colombia Médica, 42(3), 303308. Recuperado de: http://hdl.handle. net $/ 10893 / 3102$
Pinilla, E., Forero, M., \& Valdivieso, M. (2009). Servicios de salud sexual y reproductiva según los adolescentes varones (Bucaramanga, Colombia). El escenario para la salud pública desde la ciencia, 27(12), 164-168. Recuperado de: http:// www.scielo.org.co/scielo.php?script=sci arttextypid=S0120-386X2009000200007.

Rodríguez, J. (2001). Vulnerabilidad y grupos vulnerables: un marco de referencia conceptual mirando a los jóvenes. Revista Cepal, O(17), 5-61. Recuperado de: http://repositorio.cepal.org/bitstream/ handle/11362/7150/S018659_es.pdf. -

Rojas, M. (2004). Lineamientos teóricos para la estimación holística de la vulnerabilidad y el riesgo de la vivienda en la salud humana en el marco del desarrollo sustentable. Cuaderno Urbano: Espacio, Cultura y Sociedad, (4), 145-174. Recuperado de: https://dialnet.unirioja.es/servlet/ articulo? codigo $=4163676$.

Torres, G. (Ed.) (2012). Primer estudio poblacionaldesaludmental, Medellín, 20112012. Medellín: The WHO World Mental Health Survey Consortium, Universidad CES, Secretaría de Salud de Medellín, Grupo de salud mental. Recuperado de: https://www.medellin.gov.co/irj/go/km/ docs/wpccontent/Sites/Subporta1\%20 de1\%20Ciudadano/Salud/Secciones/ Publicaciones/Documentos/2012/ Investigaciones\%202011-2012/Libro\%20 Salud\%20Mental\%20-\%202011.pdf

Unicef.(2011). Estado mundial de la infancia: la adolescencia: una época de oportunidades. Recuperado de: http://www.unicef.org/ spanish/publications/index_57468.html

Vargas, A., \& García, V. (2008). Violencia urbana, seguridad ciudadana y políticas públicas: la reducción de la violencia en las ciudades de Bogotá y Medellín. Pensamiento Iberoamericano, (2), 249-270. Recuperado de: https://dialnet.unirioja.es/ servlet/articulo? codigo $=2873366$. 